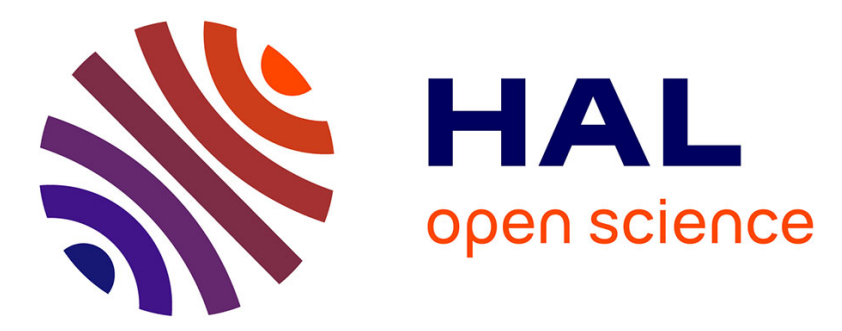

\title{
Interoperability analysis in critical collaborative processes: an application to the healthcare sector for complex patient assistance
}

\author{
Nicolas Daclin, Sihem Mallek-Daclin, Vincent Chapurlat
}

\section{To cite this version:}

Nicolas Daclin, Sihem Mallek-Daclin, Vincent Chapurlat. Interoperability analysis in critical collaborative processes: an application to the healthcare sector for complex patient assistance. Int. Journal on Emergency Management, 2016, 12 (4), pp.435-456. 10.1504/ijem.2016.079847 • hal-01930460

\author{
HAL Id: hal-01930460 \\ https://hal.science/hal-01930460
}

Submitted on 25 May 2021

HAL is a multi-disciplinary open access archive for the deposit and dissemination of scientific research documents, whether they are published or not. The documents may come from teaching and research institutions in France or abroad, or from public or private research centers.
L'archive ouverte pluridisciplinaire HAL, est destinée au dépôt et à la diffusion de documents scientifiques de niveau recherche, publiés ou non, émanant des établissements d'enseignement et de recherche français ou étrangers, des laboratoires publics ou privés. 


\title{
Interoperability analysis in critical collaborative processes: an application to the healthcare sector for complex patient assistance
}

\author{
N.Daclin ${ }^{1}$, S.Mallek-Daclin, V.Chapurlat \\ Ecole des mines d'Alès \\ Laboratoire de Génie Informatique et d'Ingénierie de Production \\ Parc Scientifique G. Besse, 30035 Nîmes cedex 1, FRANCE \\ E-mail: \{nicolas.daclin, sihem.mallek, vincent.chapurlat\}@,mines-ales.fr
}

\begin{abstract}
Developing interoperability is a major issue in collaborative processes. For instance, interoperability is crucial for collaborative processes focused on crisis or healthcare-system management. Partners have to interact trustingly and efficiently. They need to share data, knowledge, best practices, resources and skills in terms of confidence, quality of exchanges and response times. They also need to be sure of the relevance and quality of their roles and actions throughout the process to achieve a desired outcome (crisis resolution, appropriate medical surgery, etc.) In this context, it is worthwhile trying to detect and solve interoperability problems prior to the execution of the process. This work focuses on interoperability in healthcare processes for complex patient assistance (diabetes, cardiovascular accidents, etc.), where different actors need to interoperate. The purpose is to show an approach for highlighting potential interoperability problems that can occur in a given process, with the support of formal verification techniques.
\end{abstract}

\section{Keywords}

Collaborative process, interoperability requirements, interoperability analysis, healthcare system.

\section{Introduction}

Various research works in recent years focused on improving the technical methods, organization and tools for enhancing the social, medical, ethical and financial performance of health care processes for complex patient assistance [1] [2] [3]. The complex patient assistance consists of a set of activity requiring the interaction of different human means (medical, technical, social), different material means, numerous files, drugs (with possible drug interaction), therapeutic protocols to treat chronic diseases (i.e., long term) or else, complex pathologies. Precisely, in such processes, different actors work together and interact

\footnotetext{
${ }^{1}$ Corresponding author
} 
to support patients, i.e., dependent, elderly persons requiring medical, social and personal monitoring to assist them in their everyday life. These actors come from different professions (nurses, doctors, ambulance staff, specialists...). They often belong to different organizations and may be in competition (professional, liberal, private or public sector). While activities throughout the patient management process can be clearly identified, their way of performing these activities using, for instance, technical resources or drugs, and the way they comply with the medical rules and constraints to be respected, impact the patient's situation, family and work, and the specific activities of other caregivers and healthcare professionals. In that sense, it is desirable to be able (1) to describe and model the healthcare process, and (2) to detect and solve potential problems prior to its execution. Among potential problems, interoperability is a crucial aspect in processes [4] within several stakeholders are involved and share documents, knowledge, activities, resources and studying the interoperability of stakeholders can improve their capabilities to anticipate the occurrence of frequent problems [5] [6]. In the same way, it can allow stakeholders to avoid breaking the collaboration, and eliminate serious "gaps" regarding to the desired outcomes of their activities.

Thus, interoperability management in healthcare processes can be considered as beneficial in each dimensions mentioned above as it is defined and applied in various fields of application over the last two decades [7] [8] [9].

The purpose of this paper is to present and illustrate the application of an approach for highlighting potential interoperability problems in a healthcare process with the support of two formal verification techniques. Based on the fact that 1) the formalization of the processes is profitable as well as 2), the formal verification to assist stakeholders to identify possible problems (e.g. bad resource allocation) in confidence is useful, the paper attempts to provide an approach to evaluate process model from the interoperability point of view. The application of such approach is based on three major assumptions:

First, the process model is provided by an expert and is considered as complete regarding to verification objectives.

Second, the requirements' checking (e.g. requirements provided by National Authority for Health's certification and stakeholders) is performed by an external expert. The proposed approach focuses on interoperability requirements description and verification to facilitate the verification of requirements that remain difficult to verify due to complexity of the model, time to verify or potential effects of a wrong verification result. In no case the approach has the pretention to verify all requirements. 
Three, the approach focuses on organizational interoperability. Conceptual and technological barriers can be also considered and the paper presents how the corresponding requirements are described without detailing the proof technique to be applied.

Lastly, this work has been applied on various case studies such as crisis management (ISYCRI: Interoperability of SYstems in CRIsis situation, ANR-06-CSOSG). As mentioned in [33], a crisis context requires "different actors from different organizations to work simultaneously in a hurry and their ability to coordinate their actions is an essential point to reach their common goal: crisis reduction". In such a context, activities, resources, information are continuously coordinated, synchronized, exchanged and shared and the inaccurate, trustworthy and fast identification of a problem related to these interactions during the crisis resolution can lead to a degradation of the situation. In another context, but involving several actors that have to interact trustingly and detect potential problem as early as possible to implement relevant correctives actions, the here described application was carried out in a project aiming to develop a platform of services (Design of the platform of services for Home Hospitalization with the society 3G Santé) for assisting complex patient care. So, this article focuses on the use of the proposed approach on a real case study from health care systems management domain. The goal of this platform is to help healthcare stakeholders to coordinate and optimize the available means and resources to ensure global assistance. This global assistance is deployed trough a collaborative process. Thus, the care assistance is a complex field in which interoperability is fundamental for various reasons:

- Medical reasons - it is necessary to manage and to improve quality and safety of care assistance.

- Technical reasons - to generalize the use of medical technology, and share medical data.

- Organizational reasons - multiple roles and multidisciplinary or multi professional skills required for care assistance make its implementation complex.

- Standardization/legal reasons - recent laws or standards [10] [11] recommend or impose the implementation of a specific device/plan/architecture.

After this brief introduction, research works within healthcare systems engineering field are presented and discussed. The different steps of the approach deployed in healthcare sector, the related concepts and the tools used to set up the approach are presented in section 3 . Section 4 presents the application of the approach on the collaborative process. The fifth section discusses the benefits, the limitations and possible extensions of the approach. 


\section{Healthcare systems engineering using formal approaches: position}

A healthcare system is a dynamic socio-technical system with complex interactions among various services and resources, described by a set of processes. The modeling, analysis and improvement of these processes are, since recent years, a field of researches and applications. Indeed, numerous research works take an interest on the engineering of these processes to manage patient information, improve quality patient care and patient safety, or else, decrease costs and time for patient treatment. In the process analysis field, some works focus on the use of simulation and on requirements proof, more oriented on the required adherence of requirements rather than satisfaction of requirements.

For instance [12] takes an interest in the improvement of medical information system to gain time to access patient information. This research applies first Business Process Management principles by the modeling of the AS-IS process and the analysis of this model and the modeling of the TO-BE process. Then, a key metric is defined (e.g. measurement of time to notification) and assessed on the as-is process model and the to-be process model. This approach is helpful to evaluate the improvement of some performances requirements to be respected by the process. However, it does not allow to analyze other requirements that the process have to respect. In this context, this article focuses on interoperability requirements but other functional and non-functional requirement can be also analyzed using the same approach.

In addition [13] and [14] focus on the use of simulation techniques to evaluate different characteristics in various cases of healthcare processes (e.g. operations of the surgical theatres, emergency care process). Once more, process is modeled and simulated by taking into account the same hypothesis as before. The AS-IS and TO-BE models are separately executed and submitted to various scenarios. Different TO-BE models can be compared indicating the more relevant solution of process reorganization to be implemented.

Simulation is a well-known, equipped and used technique. It is done on a theoretical model whose behavior is considered to be similar to the behavior of the studied system. However, simulation is unable to assume all of the reachable system's behavioral scenarios. Then, the exploitation and the analysis of the results are difficult and require human expertise. Finally, although the main goals of research works presented hereinbefore attempt to improve healthcare processes, these did not consider other requirements than performance and particularly interoperability criteria in their analysis.

At the opposite, the approach proposed by [15] focuses on the detection and the correction of defects that can occur in a clinical blood transfusion process. This detection is based on a 
verification technique that allows to determine i.e., to prove that a requirement is respected or to obtain a counter example showing the requirement cannot be satisfied. For this, the process is modeled using finite-state automaton language. A model checker analyzes behavior i.e., the various functioning states reached by the process and determines the truth value of the requirement. Detection of defects using techniques as presented in this approach allows to verify exhaustively a process - with several requirements - quickly and accurately. Moreover, some tools allow to show a counter example in the case of a requirement is violated. However, this approach does not propose a clear methodology to collect requirements and to obtain a repository to be used at will. Then, the model of the process uses a language such as finite state automaton which is difficult to understand and to use for a non-expert in comparison with less formal process modeling language. Furthermore, this work does not mention how to point out the detected problems. Moreover, it is to note that requirements shown in this approach are not related to interoperability. Works mentioned before show the interest and the importance to manage and to improve healthcare process engineering using analysis or formal verification techniques. Each has its own advantages but does not consider interoperability requirements (especially to make a set of requirements available for stakeholders), does not allow users to model their process with a user friendly language and does not point out the detected problem. Finally, it is to note that, regardless of application domains (crisis management, industrial, healthcare...), simulation techniques are more often used than model checking to perform verification of processes. The following table presents the previous approaches regarding to the interoperability analysis in collaborative context with regards to collaborative process modeling of the collaboration, the consideration of requirements modeling and the consideration of the identification of interoperability defect.

\begin{tabular}{cccc}
\hline Approach & $\begin{array}{c}\text { Process modeling } \\
\text { consideration }\end{array}$ & $\begin{array}{c}\text { Requirement-based } \\
\text { verification }\end{array}$ & $\begin{array}{c}\text { Interoperability defect } \\
\text { identification }\end{array}$ \\
\hline$[12]$ & ++ & -- & -- \\
{$[13]$} & ++ & - & -- \\
{$[14]$} & + & - & -- \\
{$[15]$} & + & ++ & -- \\
\hline
\end{tabular}

$\overline{\text { Table 1. Comparative study of approaches regarding to the proposed approach }}{ }^{2}$

\footnotetext{
${ }^{2}$ We adopt the following notation to evaluate existing research works:

- $\quad++$ : address the issue

- $\quad+$ : relevant but partly address the issue

- $\quad$-: irrelevant to the issue

- $\quad--$ not considered
} 


\section{Approach applied to healthcare sector}

The approach and contribution presented are related to the research developed in [16]. This approach highlights potential interoperability problems that can occur in a process, with the support of formal verification techniques. The verification is performed on a model of the collaborative process. The approach consists of a series of steps from the collection of needs to the implementation of the process (Figure 1).

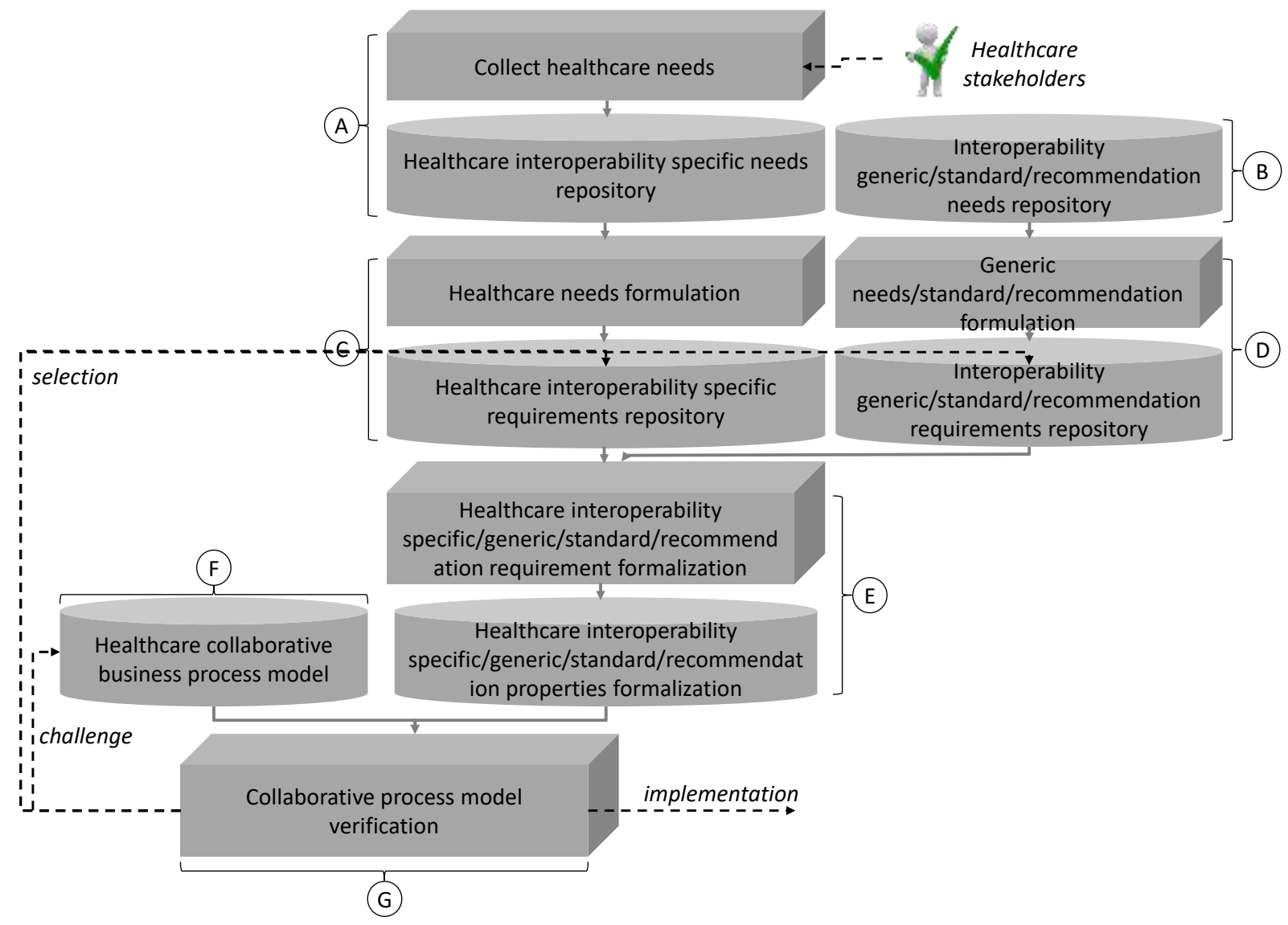

Figure 1. The approach applied to the healthcare sector (adapted from [16])

The first step consists in collecting healthcare needs through several interviews with stakeholders of various kinds, including:

- Patients and care workers: homecare worker, volunteer

- Care assistance actors: medical practitioner, pharmacist, nurse, physiotherapist, hospital department.

- Supervising authorities and financiers: health department, health insurance fund, private health organization

- Suppliers and service providers: ambulance driver, suppliers of medical devices, platforms, etc.

The objective is to get a first repository of interoperability needs related to healthcare systems (A) that means the expressed needs concern only this kind of system and cannot be apply in 
other context. These needs are strongly business-oriented and related to the interoperability of healthcare process and specifically to the process under study.. This first repository is validated, completed and improved with the help of all the stakeholders. Alongside, an other repository (B) is built and includes three kinds of requirements. First, it considers the generic needs, i.e., the interoperability needs that can be verified on any process model in any context (e.g. industrial process). Second, it includes needs stemming from standards used in healthcare domain. Lastly, it includes also needs from recommendations issued by health authorities. It is to note that if a specific need appears to be sufficiently generic to be applied on any healthcare process, it can be include in this repository for a later use. Once the needs are extracted, they are clarified and re-expressed using a formulation that avoids all the problems of natural language such as omission, ambiguity, conflict, repetition and imprecision. The result of the re-formulation is a requirement, i.e., a "statement that specifies a function, ability or characteristic that a system must satisfy in a given context" [32]. Thus, the needs belonging to the previous repositories are re-expressed to get the interoperability requirements' repositories. Moreover, as the approach aims to identify and anticipate interoperability problems, it is necessary to classify and structure identified interoperability requirements to (1) detect problems as precisely as possible, (2) enable traceability and (3) implement appropriate corrective actions. To this purpose, [17] defines a generic interoperability requirements repository based on 4 classes such as:

- Compatibility requirements, (interfacing aspects e.g. definitions of responsibilities/authorities, semantic of data...).

- Interoperation requirements, (running phase of the collaboration and performance of the interactions, e.g. resource availability, quality of exchange, exchange time).

- Autonomy requirements, (ability of stakeholders to independently maintain their autonomy - during collaboration) in terms of governance and operation. Governance autonomy means that stakeholders remain able to take their own decisions and to retain a space for decisionmaking to reach their own objectives. Operational autonomy means that stakeholders can receive or provide services while operate [18].

- Reversibility requirements (end of the collaboration i.e. when partners stop their involvement in the care assistance) check whether partners are still able to achieve their objectives after the collaboration, despite adaptations or changes.

Furthermore, for a better understanding and accuracy, a requirement is specified with the nature of the interoperability problem [5] as Conceptual (syntactic and semantic aspects of 
systems that interoperate, i.e., data model, semantic of data, expressivity...), Organizational (definition of responsibility, authorities as well as organizational structure to interact under good conditions), and Technological (use of information technologies and more precisely the standards that are used to present, store, exchange, process, and communicate data through the use of computers). All the requirements, with their classification, lead to a specific interoperability requirements repository for healthcare systems which is as exhaustive as possible $(\mathrm{C}, \mathrm{D})$.

Afterwards, two steps are required before verification. Firstly, the model of the collaborative process has to be done. In our case, the modeling language used is BPMN [20] and provides a standardized notation readily understandable by all the stakeholders involved in the design, development and monitoring of the collaborative process (F). However, BPMN does not allow to consider and to model specific concepts or attributes related to interoperability but also to healthcare sector. In this case, it is necessary to enrich this modeling language to embed interoperability requirements. The proposed conceptual enrichments described in [20] include interoperability concepts. Secondly, depending on analysis needs, the requirements must be formalized under the form of properties so that formal verification techniques can be applied (E).

Finally verification is performed on the process model, using either formal verification techniques or expertise (Figure 2) [21] [22] [23] [24] (G). The used formal techniques consider two temporal hypotheses. Firstly, a property is a-temporal i.e., independent of time and verifiable for all time of the collaboration. Secondly, a property is temporal i.e., dependent of time and verified only at some times of the collaboration. Thus, the first technique is based on Conceptual Graphs [25] [26] for a-temporal properties. The advantages are (1) it describes the collaborative process and interoperability requirements using the same language; (2) its graphical form is convenient to handle; (3) it has mathematical foundations and mechanisms [27]. The second technique is based on model checking [24] for temporal properties. The advantages are (1) it includes temporal aspects of the collaboration; (2) it considers all states of the collaborative process throughout the collaboration; (3) it gives formal proof of the problems that exist. Finally, expertise is deployed when interoperability requirements highlight particular perspectives that cannot be described due to modeling languages limitations.

The use of these verification techniques requires that the collaborative process modeled using BPMN is translated into an equivalent model allowing verification techniques [29]. The first equivalent model enables to provide proof of a-temporal properties using Conceptual Graphs 
[16] and the verification is performed using the COGITANT tool [27]. The second equivalent model, for proof of temporal properties, is obtained using a behavioral modeling language called Network of Timed Automata (NTA) [16] [28] and the UPPAAL model checker is used [24].

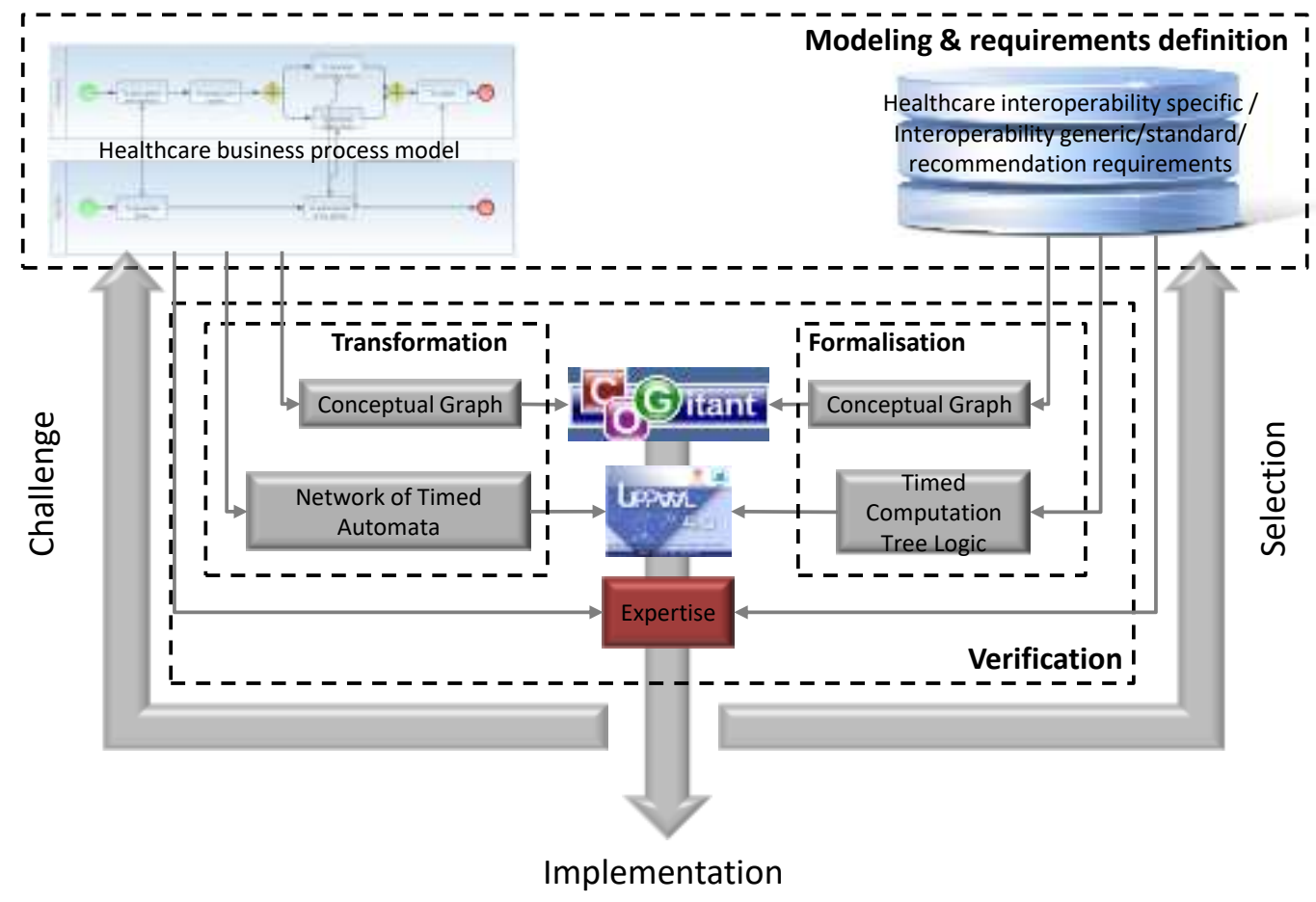

Figure 2. Verification of healthcare assistance collaboration

These transformations are based on rules defined and implemented without end-user's action. For instance, regarding to the transformation into NTA, the source is a process model conforms to the BPMN metamodel and the target is a NTA conforms to the UPPAAL metamodel which represents the process model behavior. Thus, the modeling objects in the process model have an automaton expressing their own behavior. Figure 3 gives the principle of the transformation (full transformation principles can be found in [32]). 


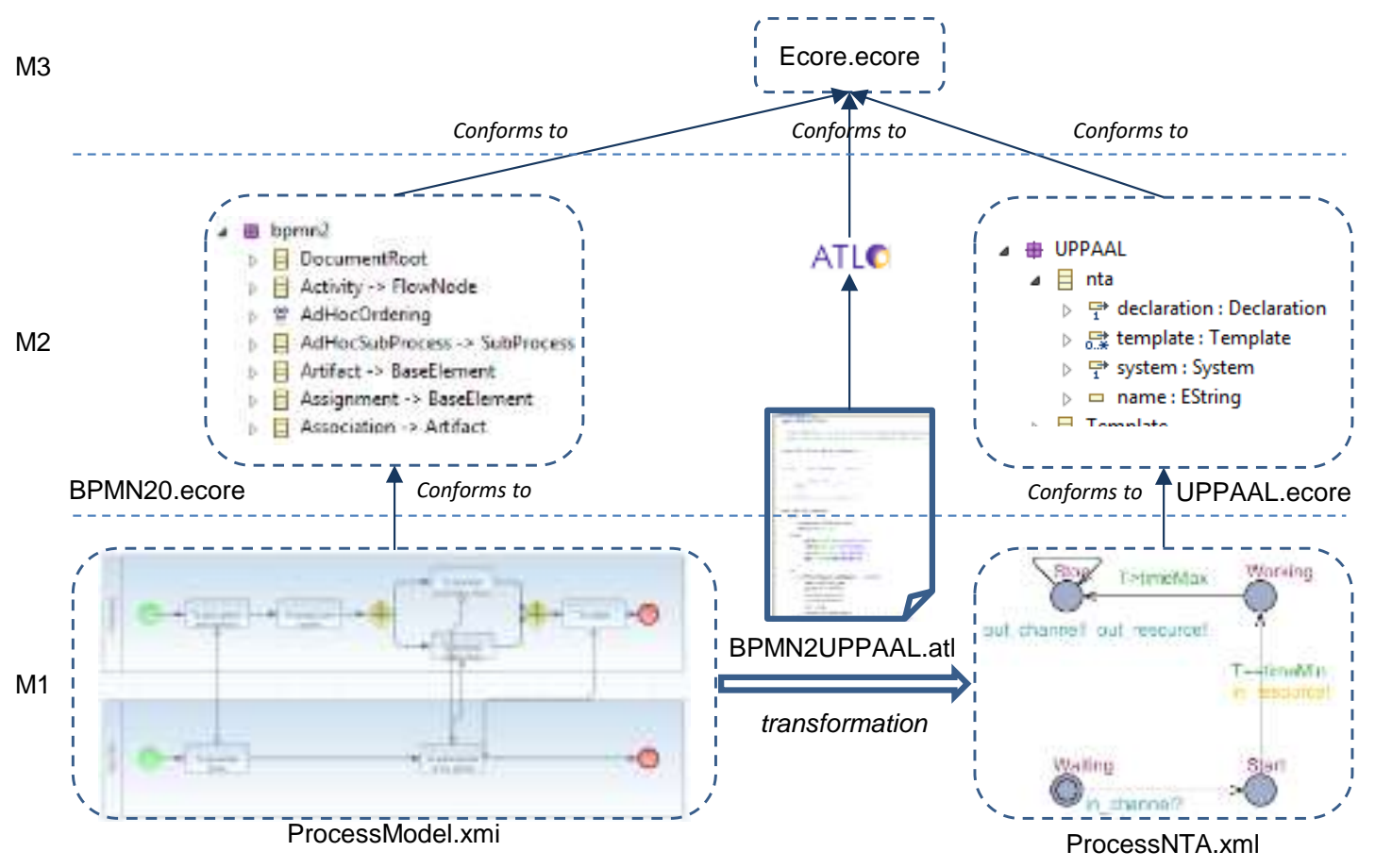

Figure 3. Principles of model transformation

Lastly, to be able to verify the interoperability requirements, they had to be formalized using Conceptual Graphs and TCTL. In addition, expertise is used to verify requirements that cannot be formally proven.

The result of the verification indicates whether or not a requirement is satisfied by the collaborative process model, highlighting the existence of an interoperability problem. Thus, if all selected requirements are satisfied by the collaborative process model, it can be implemented. Otherwise, (1) the collaborative process model is challenged to provide adequate solutions, and (2) the user can select other requirements before other verifications.

\section{Application to a collaborative process for complex patient assistance}

\subsection{Healthcare interoperability needs}

The collaborative process requires to satisfy the needs formulated by stakeholders. They are mainly related to services (provided by actors), information about care assistance, data (security, memorisation, access...), training (good practices, protocols, reference model, feedback), user friendliness and interoperability. With regard to interoperability needs, they are generic, i.e., applicable to various types of collaborative processes in various domains of expertise, or specific to process related to healthcare systems. Hereafter eight needs related to interoperability are presented:

Need 1: "The platform receives and sends document (e.g. invoice, patient file...)".

Need 2: "The platform uses and makes available some communication means between actors and persons concerned by care assistance". 
Need 3: "The platform lists and centralizes information (available resources, available places, services...) that can be necessary for the care assistance of complex patients (anticipate actions) and particular patient (fast action)".

Need 4: "The platform provides for each stakeholder access to modify or complete information for care assistance".

Need 5: "The platform coordinate and synchronize actors' activities for care assistance (health professionals, service providers and medical devices; caregivers...)".

Need 6: "The period for assessing the condition of a patient in stable condition and its operations management must be less than 48 hours (this time for a patient whose situation deteriorates significantly and becomes critical, is to be defined)".

Need 7: "The platform must reduce the cost of care for patients in the reference group, relative to non-group monitoring, reaching at least an equal quality level".

Need 8: "There is a written and signed prescription, which is complete, readable, nonambiguous and with a clear content, for all medical procedures".

For instance, the first need is generic and already existing in the generic interoperability requirements repository [21]. That means it is usable in any other domain where information are shared. Regarding to the second, third, fourth, fifth and seventh need, they could be sufficiently generic to be applied in other domains (including other healthcare processes) where a platform, authorizations, coordination... are required. Lastly, regarding to the sixth and the eighth need, they are specific to the studied health care system and are implemented in the healthcare interoperability specific needs repository.

\subsection{Healthcare interoperability requirements}

First, the needs have to be expressed in such a way as to be expurgated of the faults inherent to natural language i.e., into requirements. Furthermore, the resulting requirements are classified to highlight a particular aspect of interoperability. The requirements extracted from the needs given in the previous section are as follows (the problem considered and the originating needs are in parenthesis). The first requirements are related to compatibility. This kind of requirement is considered as invariable before process execution and is related to the interfacing between participants (e.g. interface between platform and patient).

Compatibility requirement 1 (technological - second need): "The platform provides the means of communication". It indicates that all means of communication used during care assistance are provided by the platform. .

Compatibility requirement 2 (organizational - fourth need): "The stakeholders have the necessary authorization to access the patients' data". It indicates that the care assistance 
stakeholders must be authorized to access a patient's data to complete, remove, add or modify them. This requirement also needs to be verified with regard to suppliers, patients and general practitioners.

Compatibility requirement 3 (technological - third need): "The platform includes a database which centralizes the information describing all stakeholders". All information to be shared or exchanged regarding to stakeholders about the care assistance must be stored in a dedicated data base.

The second set of requirements is related to interoperation and focuses on the ability to interact during process execution. The veracity of these requirements is considered variable during the collaboration in function of interactions between participants.

Interoperation requirement 1 (organizational - first need): "For each datum received, a receipt is sent". It indicates that a receipt has to be sent in response to all data (e.g. invoices) received by a partners during the collaborative process. The use of a receipt mechanism ensures that partners receives and sends datum really.

Interoperation requirement 2 (organizational - seventh need): "The cost of care assistance is less than the cost of ordinary assistance". It indicates that the implementation of a platform for care assistance must reduce the cost of care assistance compared to ordinary assistance. .

Interoperation requirement 3 (organizational - sixth need): "The period between the beginning of the activity "initialize platform" and the activity "initialize care assistance" must be less than 48 hours". In the case of a patient in a stable condition, the time lapse between the evaluation of the state of a patient and effective assistance must be less than 48 hours. .

Interoperation requirement 4 (organizational - fifth need): "Actors' activities are coordinated". It indicates that all activities performed by actors during care assistance must be coordinated. For instance, the implementation of a given therapeutic protocol involving different health experts and different drugs with possible harmful interactions, etc. requires considerable coordination to perform the process safely.

Interoperation requirement 5 (conceptual - eighth need): "Each medical procedure matches a written prescription". It is to note that other requirements can be extracted from this need, showing the importance to re-express needs into requirements for their future verification. For instance, "each medical procedure matches a signed prescription" or "a prescription is readable" and so on, are requirements coming from the same need.

Finally, it is to note that no requirements related to autonomy and reversibility are defined from the needs. 


\subsection{Healthcare interoperability properties}

Depending on their temporal nature, requirements must be formalized. In this case, a-temporal requirements are formalized using Conceptual Graphs and Temporal requirements are formalized using temporal logic (TCTL). For a better understanding of the formalization process and the use of the verification techniques, a brief introduction to the principles of Conceptual Graphs and TCTL is proposed.

Conceptual Graphs verification is done with mathematical mechanisms called projection. It consists in the projection of a constraint on the Conceptual Graph representing the model of the process. Two kinds of projection are executed: positive (projection of a cause and a conclusion) or negative (projection of a simple graph) [27]. The projection mechanism is the projection of a given requirement translated into a Conceptual Graph (property), on a Conceptual Graph that represents the model of the process.

For instance, the first compatibility requirement is modeled using a Conceptual Graph in Figure 4. It means (compatibility requirement 1) that a participant in the process corresponds to the platform (participant has name platform) and has a resource such as communication device. It is made up of a cause and a conclusion. This requirement will be satisfied if (1) the cause is projected on the Conceptual Graph that represents the process model, and (2) the requirement can be fully projected (cause and conclusion) on the process model.

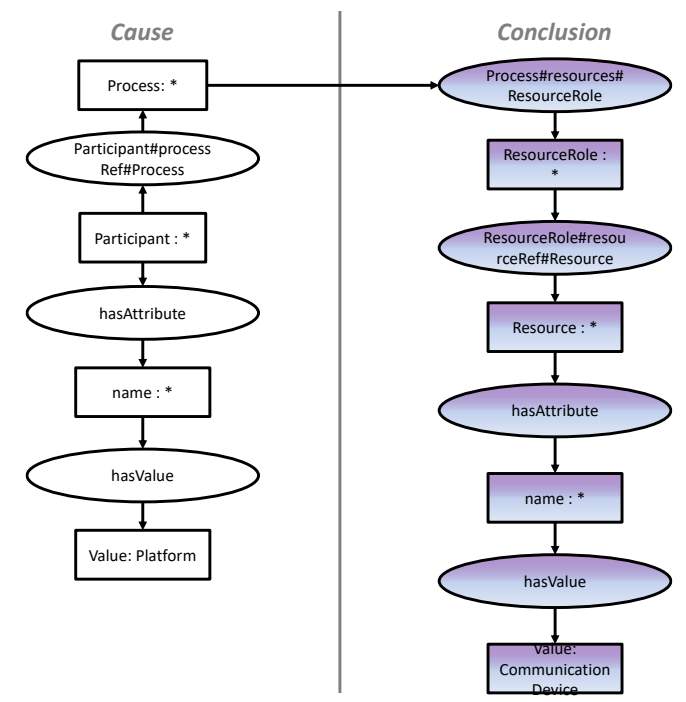

Figure 4. Example of a requirement modeled with a conceptual graph

Verification with the model checker uses TCTL to consider several possible futures based on the state of a system and relies on modalities (e.g. it is possible to reach a state in which the property is satisfied). The model checker exhaustively verifies properties through all the reachable execution paths of the behavioral models. For instance, the third requirement is modeled as follows: 
E<> InitializePlatform_.timeMax - InitializeCareassistance_timeMin $<48$

It will be satisfied if it exists a path in the process where the difference between the end time of the activity "Initialize platform" and the start time of the activity "Initialize care assistance" is less than 48 time units (hours in this case). The compatibility requirements presented hereafter have a-temporal aspect and are formalized into properties with positive constraints using Conceptual Graphs (Figure 5). 


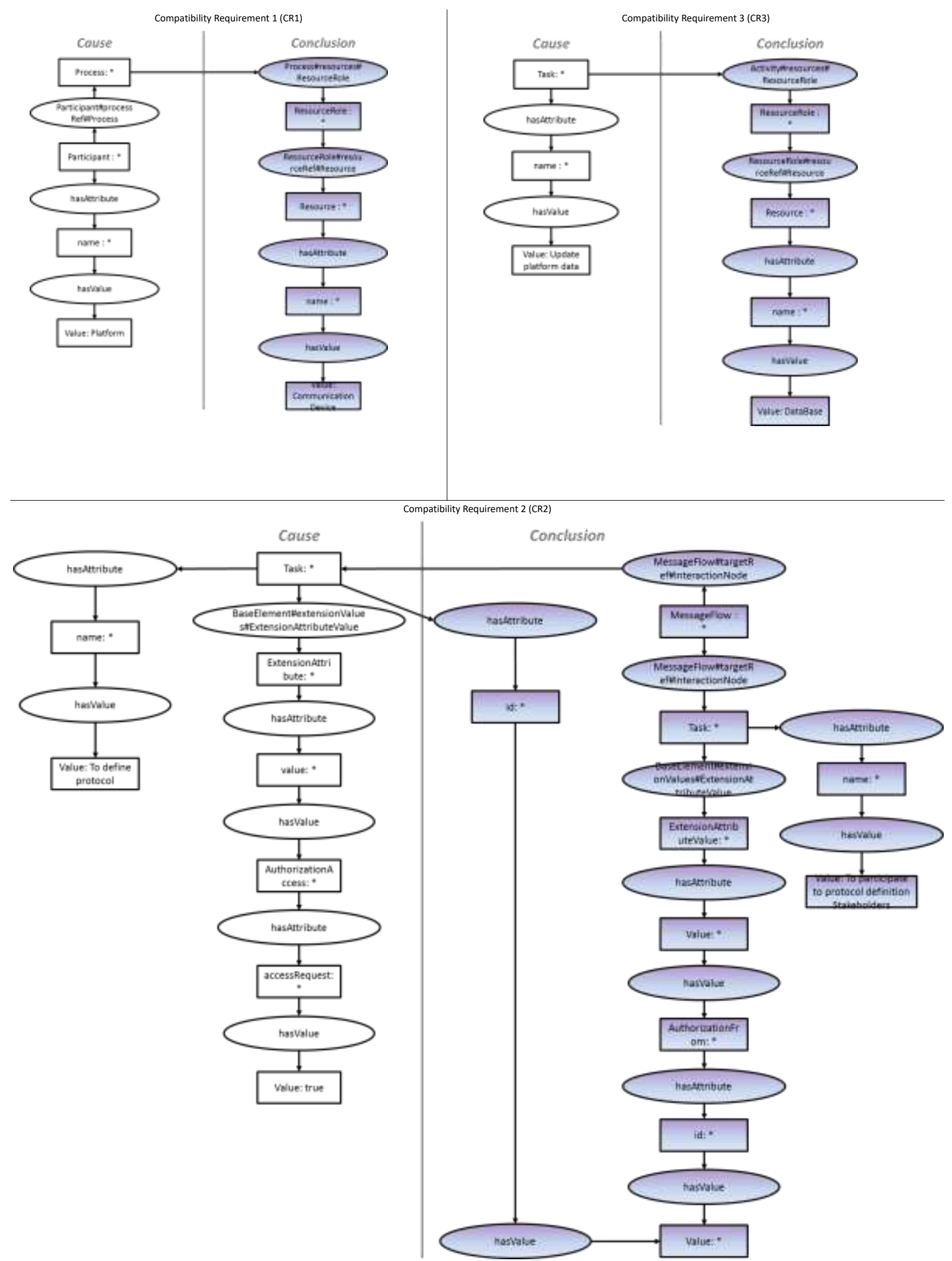

Figure 5. Compatibility requirements formalized using Conceptual Graphs

The interoperation requirements presented hereafter have both a-temporal and temporal aspects. 


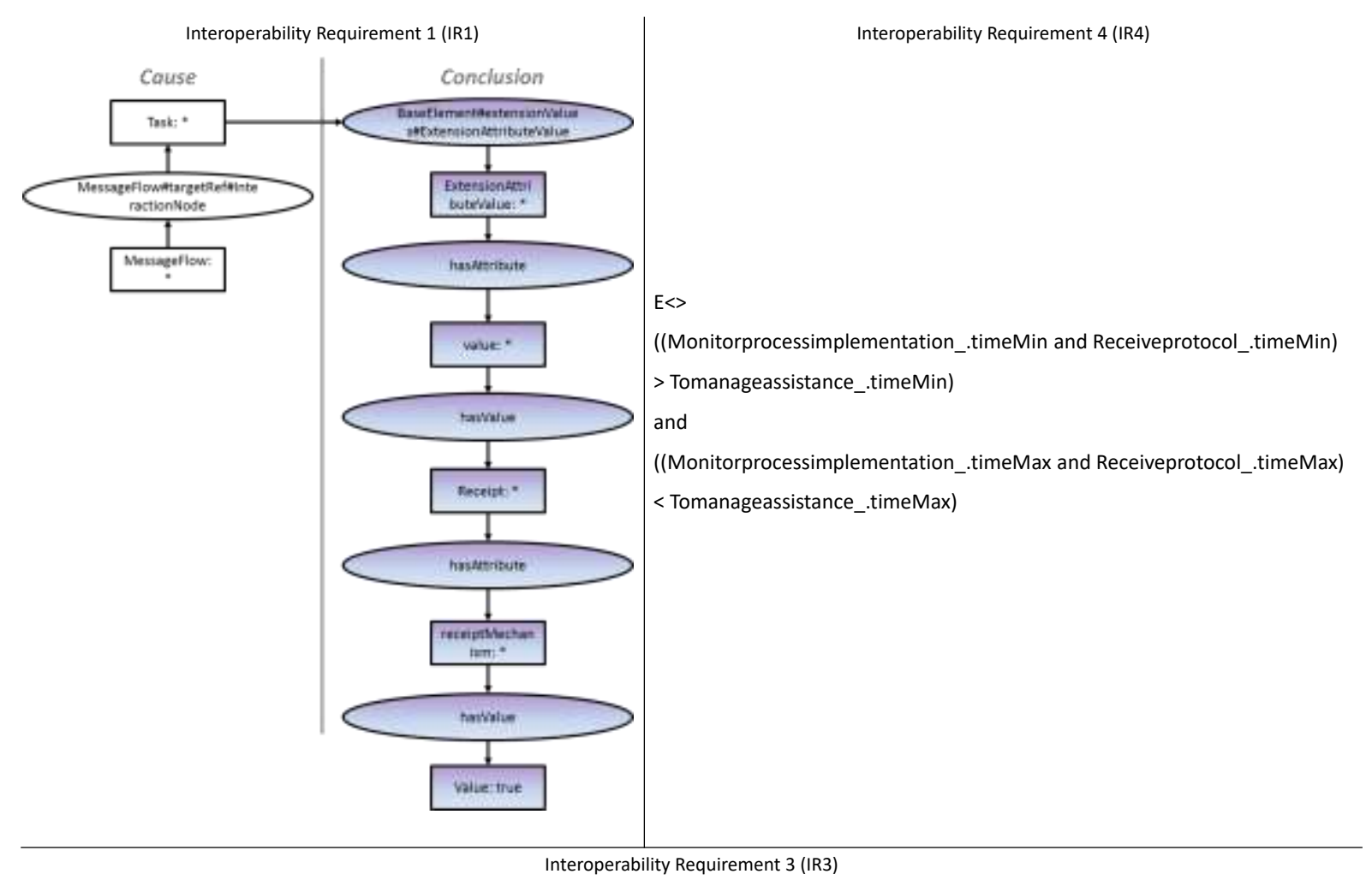

E<> InitializePlatform_.timeMax - InitializeCareassistance_.timeMin $<48$

Figure 6. Interoperation requirements formalized using TCTL

For a better understanding, interoperation requirements IR4 can be interpreted as follows: "it exists a path in the process within the activities to monitor process implementation and to receive protocol are executed between the start date and the end date of the activity to manage assistance". Finally, the second and the fifth interoperation requirements (IR2 and IR5) cannot be verified on the process model because their concepts are not included. These requirements are verified using the expertise technique.

\subsection{Healthcare collaborative process model $^{3}$}

The process model set up five types of participants: platform, stakeholders (except for medical practitioners), suppliers, patients and general practitioners.

The platform is "responsible" for assisting health actors, coordinating, and optimizing means and resources to ensure care assistance from its initialization to its end. It must therefore deal with all data related to the care assistance process (patient medical files, incident cards, situation reports, protocol...). These data are collected and stored in a database and shared by participants. They are also updated (creation, modification, cancelling) according to defined authorizations and with a dedicated software. The platform also implements all care assistance tasks, including critical steps such as:

\footnotetext{
${ }^{3}$ A demonstration is available at: goo.gl/TBYHk1.
} 
- The initialization of the care assistance. It initializes the patient file, evaluates the state and situation of the patient and decides whether the patient is eligible for care assistance. It specifies a classical therapeutic protocol; validates the availability and capacity of the protocol, devices and stakeholders.

- The definition of the therapeutic process. It defines/modifies (feedback coming from the management of process implementation) the objective of the protocol, plans the actions of the care assistance, and optimizes resources.

- The management of process implementation. It launches the therapeutic protocol, and evaluates (1) the conformity of the application regarding the description of the previously defined protocol, and (2) the situation and state of the patient. This activity also analyzes the difference between the objective of the protocol and the real situation of the patient and can request protocol modifications.

The stakeholders, suppliers, patients and medical practitioners are involved in the care assistance through the use of the platform. More precisely, these participants are highly involved in defining the therapeutic process to be implemented and managing the implementation of the therapeutic process. All message flows between the activities from different participants (e.g. between To define protocol and Define protocol) represents an interaction (e.g. exchange of information). The full process model where interoperability requirements will have to be verified is given in figure 7 .

Then, the verification of an interoperability requirement is closely related to the model used for the collaborative process and the interoperability concepts that embedded in this model. Although a given modeling language offers the possibility of reaching a full model for a specific field, it may require adaptations to be deployed in another field. This is the case of BPMN, perfectly adapted to the Information and Technology context, but which suffers lacks in other contexts (as interoperability in healthcare process). This means that enrichments of the modeling language must be performed to consider interoperability concepts and furthermore healthcare concepts. Thus, depending on the context in which the approach is applied, this enrichment can be carried out in two ways: conceptual and operational. Conceptual enrichment consists in adding attributes to modeling elements. For instance, the interoperation requirement IR4 considers the start date and the end date of the activity "to manage assistance". However, BPMN does not allow specifying these two attributes on an activity. It is necessary to enrich the language to consider these attributes and furthermore to verify the requirement. The result of this enrichment allows actors to model their collaborative process according to the context concerned, as well as to collect a maximum 
amount of knowledge for elements involved in its execution and related to interoperability. This allows, as well, the use of formal verification techniques such as model checking.

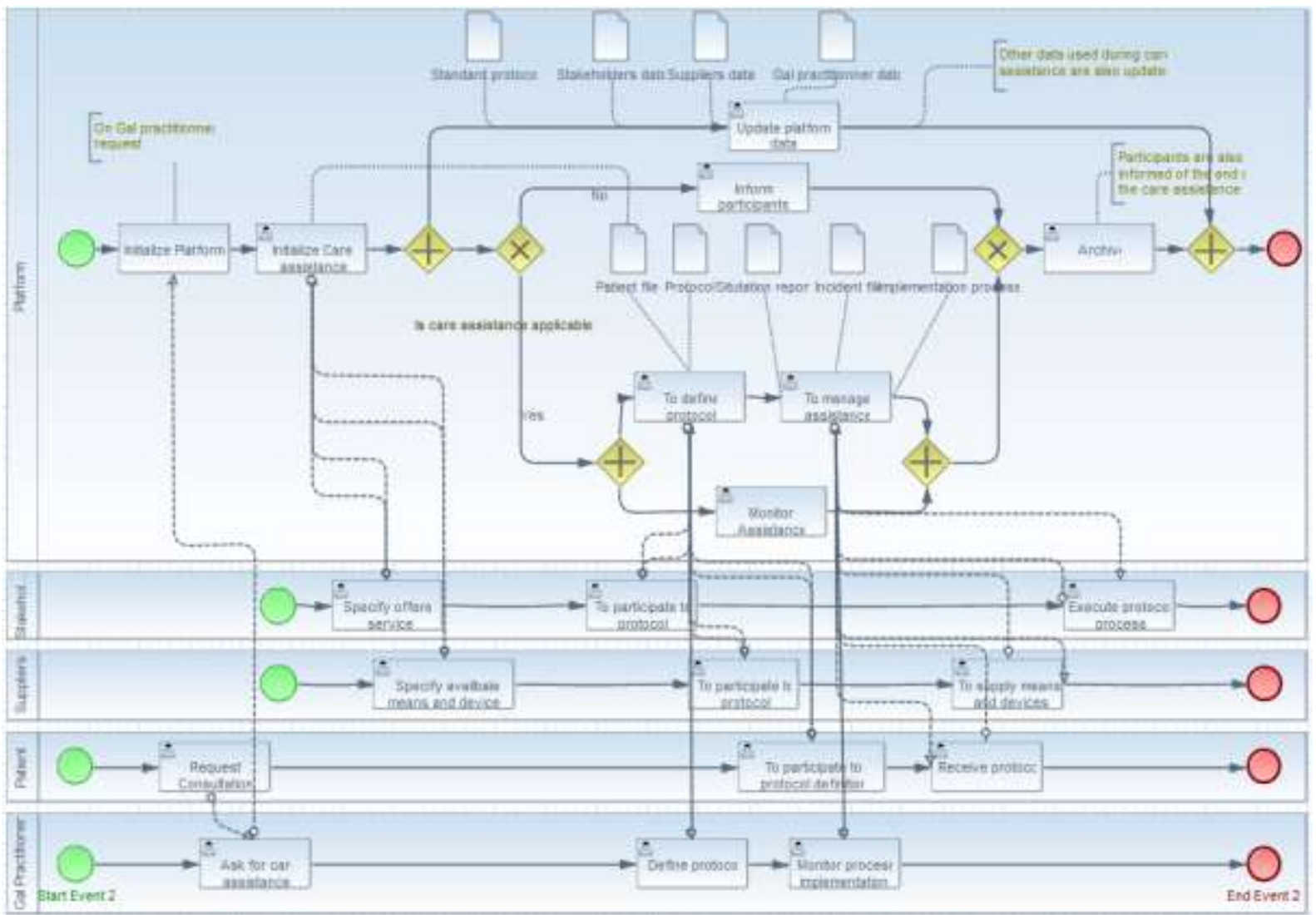

Figure 7. Business process model of the platform of complex patient care assistance

4.5 Verification of the healthcare business process model and analysis of results

Once the model is performed, the stakeholders need to select a set of requirements previously expressed in conceptual graph and/temporal logic by an expert in formal verification. To this purpose, a set of requirements that formally express those presented in section 4.2 - but always in natural language to be understandable - are made available. They are selected and instantiated with information coming from the process model. For instance, the formal requirement "if task exists then resource is used » coming from the compatibility requirement 3 is instantiated with the name of tasks and resources deployed in the process. Thus, the stakeholder can build the requirement "if Update platform data exists then DataBase is used" to make sure that the platform really use a database when information are updated. The following figure shows the principle of the selection and instantiation of a requirement with the aim of its verification. The set of information that clarifies the requirement (nature, i.e., Temporal/A-temporal, type, i.e., Organizational, Technological..., verification technique, i.e., model checking, conceptual graph) is given to the stakeholders. 


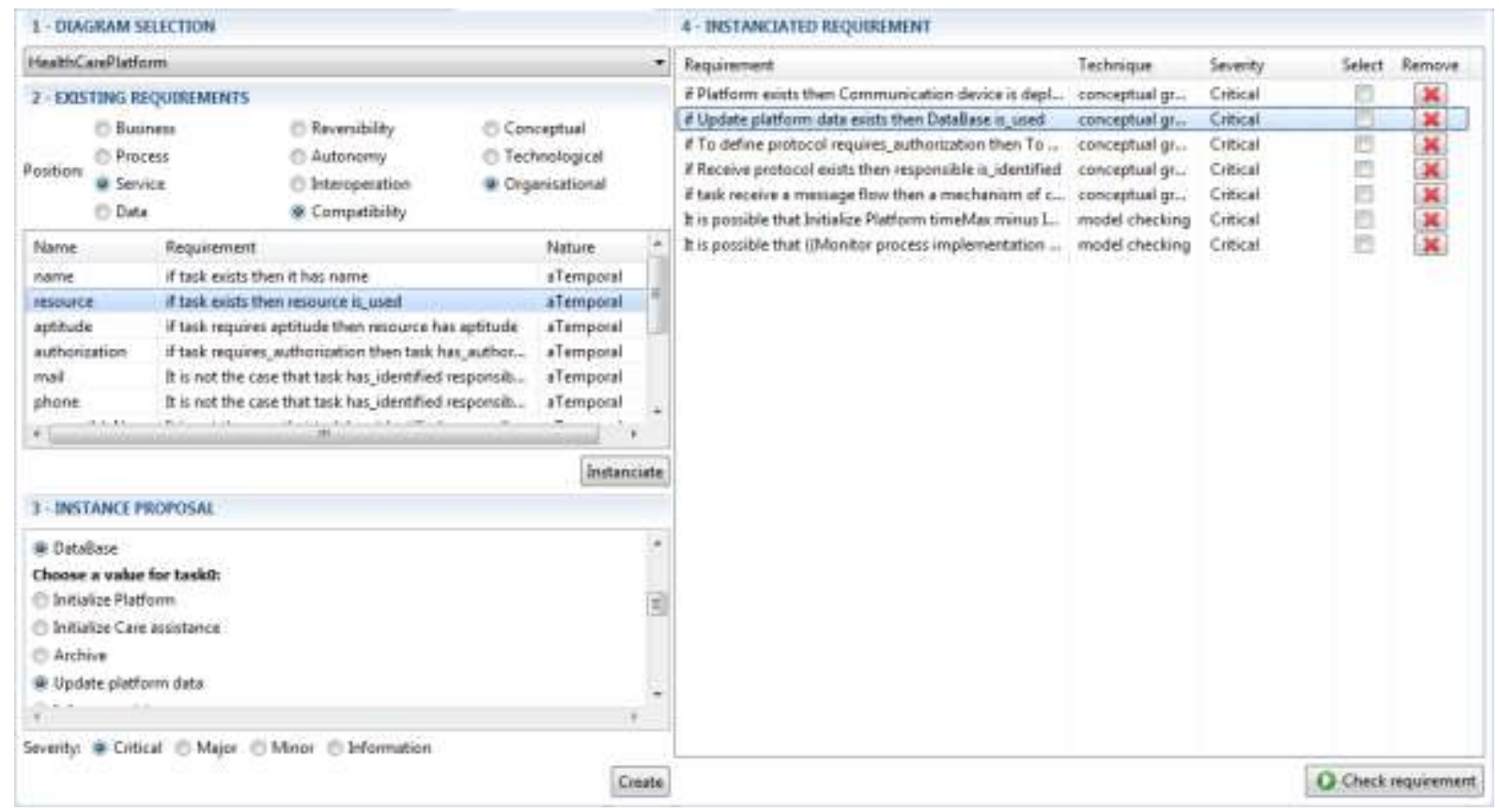

Figure 8. Selection and instantiation of requirement by the stakeholders

Then, the verification is performed and the results are brought to stakeholders' attention. The process model is transformed into a conceptual graph and a NTA in order to apply both kinds of requirements. These transformations and the verification are automated without other handling for the end-users. Lastly, let's note that the Conceptual Graph that represents the requirement "if Update platform exists then DataBase is used" is given in figure 5 (Compatibility Requirement 3). Figure 9 shows the results of the verification of interoperability requirements on the healthcare process model. 


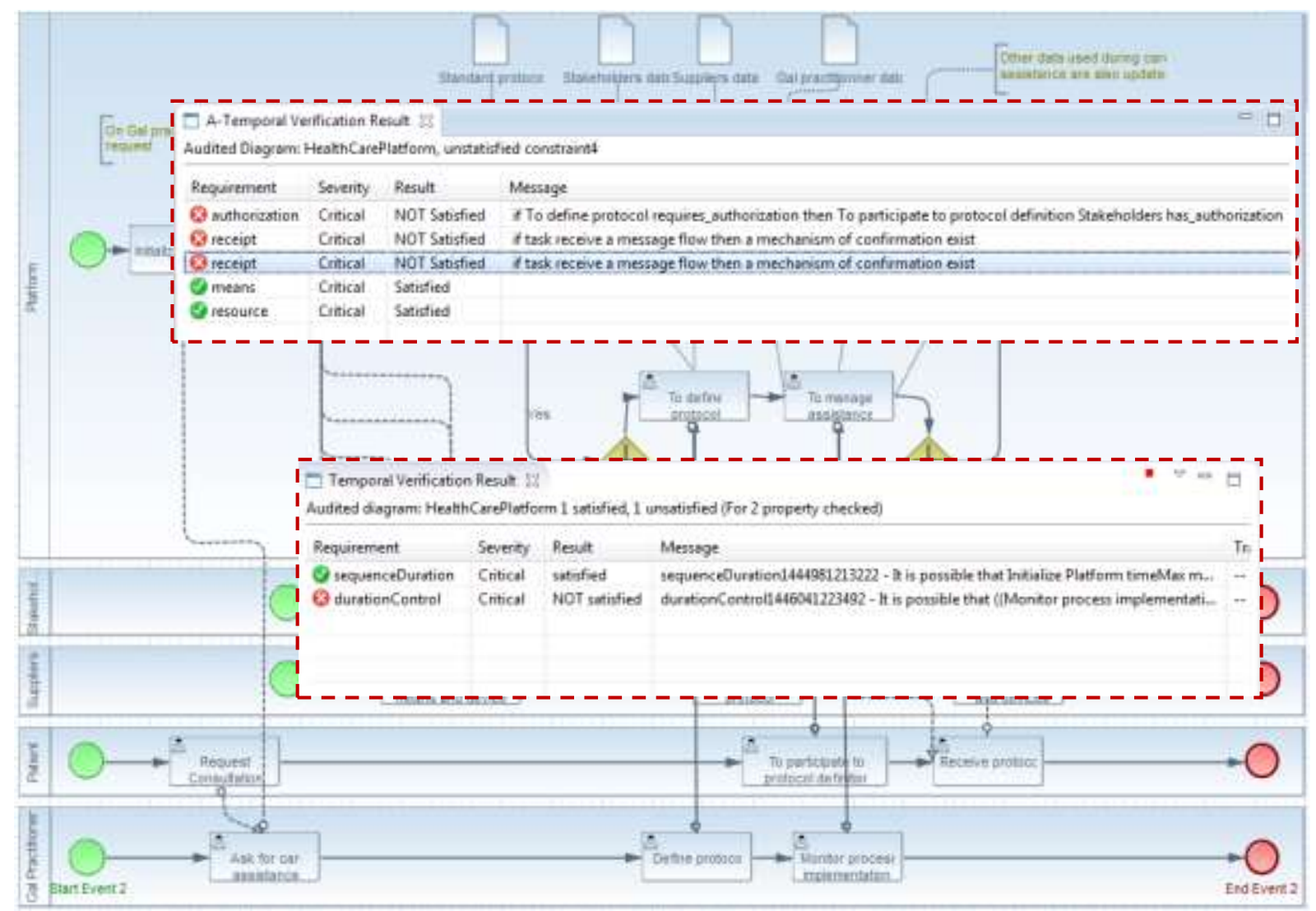

Figure 9. Results of the interoperability requirements verification

The results indicate that the compatibility requirements "CR3 stakeholder authorization" and the interoperation requirements "IRI receipt mechanism" and "IR4 coordination" are not satisfied. For instance, the Compatibility requirement CR3 indicates that stakeholders do not have the authorization to access data as required to participate to the definition of the protocol. Without this authorizations stakeholder cannot participate efficiently to the building of the appropriate therapeutic protocol. Indeed, the non respect of this requirement leads to:

- A loss of time (1) to establish or modify the protocol, (2) to find and to exchange/share the right datum - with other actors - to defines a protocol outside the collaborative process;

- A deterioration of the quality of the protocol due to the risk of exchange of wrong information (bad optimization of resources, unavailable resources, wrong patient information, non ability of a given resource...) and, more than anything;

- A possible harmful impact on the patient in terms of care assistance that can lead to a serious degradation of its situation.

Finally, the verification of the last requirement (IR 2) is performed by expertise and not considered here. The problems highlighted for each unsatisfied requirement can be corrected before the care assistance becomes effective and it is possible to perform the verification until all selected interoperability requirements are satisfied. 


\section{Discussion about the use of the approach}

The main objective of the approach is to show the applicability and the contribution of the process modeling and formal methods to the management of collaborative process and interoperability. It can be difficult to extract and re-express a right requirement(s) from a need formulated by all the stakeholders. These difficulties come from the natural language (omission, ambiguity...) but also from the specificities of the business language used within a specific field (healthcare in the application). Work such as the OMG SBVR standard [30] can be used to make the requirement writing process easier. In this way, the purpose of a limited but formal and sufficient business language can allow to express requirements, faster and more efficiently. Recent works presented in [31] propose to use a dedicated language that helps actors to write their own requirements to be verified.

A specific interoperability requirement repository regroups all the requirements identified by stakeholders. It can be improved by stakeholders themselves to be as exhaustive as possible and relevant to the needs related to their domain. Furthermore, the generic interoperability requirement repository - independent from the studied domain - must be regularly updated with new generic requirements. Hence, the formalization of requirements, into properties, using Conceptual Graphs and TCTL, may appear time-consuming and difficult (e.g. modeling of Conceptual Graphs). However, once requirements have been formalized they can be incorporated in a repository (modifiable, add-on, removal) that can be re-used at will, according to needs analysis, the process model, etc.

Formal verification can be used as a complement to human expertise when it is possible. Indeed, formal verification can remedy some gaps and problems inherent to human expertise. Human expertise requires considerable skill, and verification based on this technique can lead to the misinterpretation of problems, non-identification of problems (complex process model, decomposition of activity into sub process, understanding of the process), incorrectly identified problems (e.g. model checking can give a counter example if a given requirement is not verified), the time required to identify problems, and the impossibility to identify timerelated problems. In that sense, this kind of approach can be a support, particularly in processes such as healthcare or crisis management which require a special attention from actors and for which the time to react and to implement relevant corrective actions must be reduced or, at least, under control.

Lastly, the use and the success of the approach depend on several considerations. First, it depends strongly on the process model which must be well formed in accordance with modeling rules, so that the transformations and verification can be applied. Indeed these rules 
- especially to get a NTA that can be executed - are based on the BPMN modeling rules and without a well formed model, verification cannot be performed. Then it depends on the information included into the model. The requirements are based on information coming from the model and populated by the stakeholders (duration, name, resource...). Without or with partial information, requirements cannot be expressed and verified. Lastly, it depends on allowed transformations. To this end, new transformation rules are regularly tested and implemented to allow the modeling of more complex process models.

\section{Conclusion and prospects}

In a collaborative context, interoperability is becoming important, especially in critical collaborative processes such as in healthcare sector. The application presented shows the use of formal techniques to highlight potential interoperability problems as quickly as possible and with confidence. Furthermore, the use of the approach before process execution allows partners to avoid common problems that could occur during implementation. Indeed the approach allows to identify problems of interoperability on a process model, by using verification techniques and offers an indication 1) on the nature of interoperability problem (conceptual, technological, organizational) and 2), on the moment at which the problem can occur (interfacing phase of partners, execution phase, disassembling phase). The approach allows also to examine the interoperability solutions to be deployed. Indeed, the indication of the identified problem can be used to find an adapted solution. For instance the interoperability framework such as proposed in [5] provides a set of interoperability solutions according to the nature of problems. Then, some interoperability requirements verifications are not considered by the approach (e.g. interoperation requirement 2). As a consequence, we propose using complementary verification techniques such as simulation based on distributed multi-agent systems to improve interoperability problem detection. The approach has also to consider the space of solutions to propose appropriate solutions to the highlighted problems. Lastly, even though the approach focuses on the interoperability requirements, it is to note that it can be used to develop other categories of requirements (e.g. functional, interface...). In that case, the process model should be extended to embed attributes and information to express these kinds of requirements and equivalent models (into the targeted verification models) should be developed and automated.

\section{Acknowledgement}

The authors thank and acknowledge all the stakeholders and the Dr. Hervé Garcin, head of the society $3 \mathrm{G}$ Santé, for their support and their contributions to the ARMINES project "Design of the platform of services for Home Hospitalization". 


\section{References}

1. R. Kopach-Konrad, M. Lawley, M. Criswell, I. Hasan, S. Chakraborty, J. Pekny, B. N. Doebbeling, Applying Systems Engineering Principles in Improving Health Care Delivery, Journal of General Internal Medicine, vol. 22, Supplement 3, pp. 431-437, 2007.

2. H.Steg, H.Strese, C.Loroff, J.Hull, S. Schmidt, Europe Is Facing a Demographic Challenge Ambient Assisted Living Offers Solutions, Ambient Assisted Living European Overview, March 2006.

3. J.C.Augusto, P.McCullagh, Ambient Intelligence: Concepts and applications, Computer Science and Information Systems / ComSIS 2007, vol. 4, br. 1, str. 1-26, 2007.

4. J. Touzi, F. Bénaben, H. Pingaud, Collaborative Information System Design: From Process Model to Information System Model. Proceedings of the 12th IFAC Symposium on Information Control Problems in Manufacturing (INCOM'2006), May 17-19, 2006, Saint Etienne, France, Elsevier, 2006.

5. ISO/DIS 11345-1: Advanced automation technologies and their applications. Part 1: Framework for enterprise interoperability, 2009.

6. IEEE, IEEE standard computer dictionary: a compilation of IEEE standard computer glossaries, 1990.

7. N. Daclin, D. Chen, B. Vallespir, Developing enterprise collaboration: a methodology to implement and improve interoperability, Enterprise Information Systems, DOI: 10.1080/17517575.2014.932013, 2014.

8. S. Truptil, F. Benaben, H. Pingaud. Collaborative process design for Mediation Information System Engineering. 6th International ISCRAM Conference - Gothenburg, Sweden, May 2009.

9. Department of Defense (DoD), Department of defense Dictionary of Military and Associated Terms, United States of America Department of Defense, 2001.

10. HL7 (Health Level seven International). http://www.hl7.org/index.cfm (last visited, October, 2015), 2012.

11. HAS (Haute Autorité de Santé). Certification manual for healthcare organisations, http://www.has-sante.fr/portail/upload/docs/application/pdf/2015-

07/certification_manual_for_healthcare_organisations_v2010.pdf (last visited, January 2016, more recent version in French at: http://www.has$\underline{\text { sante.fr/portail/jems/r_ 1439924/en/manuel-de-certification-des-etablissements-de-sante- }}$ v2010-revise-avril-2011), 2008. 
12. J. Becker, R.Fischer, C. Janiesch, H. J. Scherpbier, Optimizing U.S. Healthcare Process: A case Study in Business Process Management. Proceedings of the thirteenth conference on information systems, Keystone, Colorado, USA, 2007.

13. A. Kumar, L. Ozdamar, Business Process Reengineering at the Hospitals: A Case Study at Singaprore Hospital. Proceedings 18th European Simulation Multiconference, Graham Horton (C) SCS Europe, ISBN 3-936150-35-4 (book), 2004.

14. S. J. Shim, A. Kumar. Simulation for emergency care process reengineering in hospitals. Business Process Management Journal, vol. 16, no 5, pp.795-805, 2010.

15. L. J. Osterweil, G. S. Avrunin, B. Chen, L. A. Clark, R. Cobleigh, E. A. Henneman P. L. Henneman, Engineering Medical Processes to Improve Their Safety: An Experience Report. Situational Method Engineering: Fundamentals and Experience, IFIP - The International Federation for Information Processing, vol. 244, pp. 267-282, 2007.

16. S. Mallek, N. Daclin, V. Chapurlat. The application of interoperability requirement specification and verification to collaborative processes in industry, Computers in Industry 63, pp. 643-658, 2012.

17. S. Mallek, N. Daclin, V. Chapurlat. Formalisation and Verification of Interoperation Requirements on Collaborative Processes. 18th IFAC World Congress (IFAC'11), Milano, Italy, 2011.

18. D. Chen, M. Dassisti, B. Elvesæter. Enterprise interoperability framework and knowledge corpus - final report. Interop deliverable DI.3, may 2007.

19. Open Management Group (OMG). Business Process Model and Notation (BPMN) version 2.0, available online at: http://www.omg.org/spec/BPMN/2.0/ (last visited, October 2015), January 3rd 2011.

20. M. Roque, V. Chapurlat, Interoperability in collaborative processes: requirements characterisation and proof approach. PRO-VE'09, 10th IFIP Working Conference on VIRTUAL ENTERPRISES. Thessaloniki, Greece, 7-9 October, 2009.

21. O. Balci, W. Ornwsby, Expanding our horizons in verification, validation and accreditation research and practice. 2002 Winter Simulation Conference, E. Yücesan, C.H.Chen, J. L. Snowdon, and J. M. Charnes Ed.,(Editors), 2002.

22. M. Edmund, Jr. Clarke, O. Grumbereg, A.P. Doron, Model checking. The MIT Press, 1999.

23. B. Bérard, M. Bidoit, A. Finkel, F. Laroussinie, A. Petit, L. Petrucci, Ph. Schnoebelen, P. McKenzie, Systems and Software verification: model checking techniques and tools. Springer, 2001. 
24. G. Behrmann, A. David, K. G. Larsen, A tutorial on Uppaal. Department of Computer Science, Aalborg University, Denmark, 2004.

25. Sowa, J.F.: Conceptual Graphs. IBM Journal of Research and Development, (1976)

26. V. Chapurlat, B. Kamsu-Foguem, F. Prunet, Enterprise model verification and validation: an approach. Annual Review in Control, vol. 27, Issue 2, pp 185-197, 2003.

27. Genest, D.. COGITANT Reference Manual version 5.2.6. 2010, available online at: http://cogitant.sourceforge.net/ (last visited, October 2015), 2010.

28. V. Gruhn, R. Laue, Using Timed Model Checking for Verifying Workflows. Computer Supported Activity Coordination 2005: 75-88, 2005.

29. ATLAS Groupe INA \& INRIA Nantes, ATL Atlas Transformation Language. Specification of the ATL Virtual Machine. Version 0.1, 2005.

30. Open Management Group (OMG). Semantics of Business Vocabulary and Business Rules (SBVR) $\quad-\quad$ version 1.0, available online at http://www.omg.org/spec/SBVR/1.0/PDF (last visited, October 2015), January 2008.

31. S. Mallek, N. Daclin, V. Chapurlat, B. Vallespir. Requirements formalization for systems engineering: an approach for interoperability analysis in collaborative process model, workshops e co-located with IWEI 2013, Enschede, The Netherlands, march 26, 2013.

32. S.J. Scucanec, J.R. Van Gaasbeek. A day in the life of a verification requirement, U.S Air Force T\&E Days, Los Angeles, California, February 2008.

33. F. Bénaben, C. Hanachi, M. Lauras, P. Couget, V Chapurlat, A metamodel and its ontology to guide crisis characterization and its collaborative management. Proceedings of the $5^{\text {th }}$ International ISCRAM Conference, Washington, DC, USA, 2008. 